\title{
An Acute Immune Response to Middle East Respiratory Syndrome Coronavirus Replication Contributes to Viral Pathogenicity
}

Laura J. Baseler, ${ }^{\star \dagger}$ Darryl Falzarano, ${ }^{*}$ Dana P. Scott, ${ }^{\ddagger}$ Rebecca Rosenke, ${ }^{\ddagger}$ Tina Thomas, ${ }^{*}$ Vincent J. Munster, ${ }^{*}$ Heinz Feldmann, ${ }^{*}$ and Emmie de Wit*

From the Laboratory of Virology, * and the Rocky Mountain Veterinary Branch, ${ }^{\ddagger}$ Division of Intramural Research, National Institute of Allergy and Infectious Diseases, NIH, Rocky Mountain Laboratories, Hamilton, Montana; and the Department of Comparative Pathobiology, ${ }^{\dagger}$ Purdue University, West Lafayette, Indiana

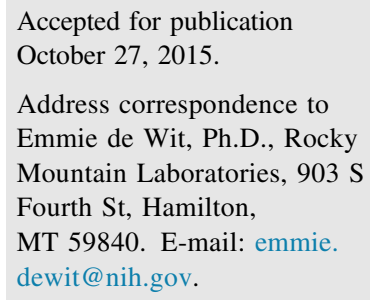

\begin{abstract}
Middle East respiratory syndrome coronavirus (MERS-CoV) was first identified in a human with severe pneumonia in 2012. Since then, infections have been detected in $>1500$ individuals, with disease severity ranging from asymptomatic to severe, fatal pneumonia. To elucidate the pathogenesis of this virus and investigate mechanisms underlying disease severity variation in the absence of autopsy data, a rhesus macaque and common marmoset model of MERS-CoV disease were analyzed. Rhesus macaques developed mild disease, and common marmosets exhibited moderate to severe, potentially lethal, disease. Both nonhuman primate species exhibited respiratory clinical signs after inoculation, which were more severe and of longer duration in the marmosets, and developed bronchointerstitial pneumonia. In marmosets, the pneumonia was more extensive, with development of severe airway lesions. Quantitative analysis showed significantly higher levels of pulmonary neutrophil infiltration and higher amounts of pulmonary viral antigen in marmosets. Pulmonary expression of the MERS-CoV receptor, dipeptidyl peptidase 4, was similar in marmosets and macaques. These results suggest that increased virus replication and the local immune response to MERS-CoV infection likely play a role in pulmonary pathology severity. Together, the rhesus macaque and common marmoset models of MERS-CoV span the wide range of disease severity reported in MERS-CoV-infected humans, which will aid in investigating MERS-CoV disease pathogenesis. (Am J Pathol 2016, 186: 630-638; http://dx.doi.org/10.1016/j.ajpath.2015.10.025)
\end{abstract}

Middle East respiratory syndrome coronavirus (MERS-CoV) was first isolated in 2012 from a human with fatal acute pneumonia in Saudi Arabia. ${ }^{1}$ Since the initial case, $>1500$ human cases of MERS-CoV infection have been detected (World Health Organization, http://www.who.int/csr/don/30september-2015-mers-saudi-arabialen, last accessed October 9, 2015); most of these cases have occurred in or near the Arabian Peninsula (Centers for Disease Control and Prevention, http://www.cdc.gov/coronavirus/mers/about/index. html, last accessed October 9, 2015). Dromedary camels, common in the Arabian Peninsula, are thought to serve as a reservoir for MERS-CoV ${ }^{2}$ which may, in part, help explain the clustering of human MERS-CoV infections in this geographic location. The exact route of transmission of MERS-CoV from camels to humans has not been definitively identified, although dromedary camels infected with MERS-CoV have been shown to secrete high amounts of infectious virus in their nasal discharge ${ }^{3}$ and viral RNA has been detected in their milk.

MERS-CoV causes a wide range of disease severity in infected humans, spanning from asymptomatic to severe,

Supported by the National Institute of Allergy and Infectious Diseases, NIH Intramural Research Program.

Disclosures: None declared.

Current address of L.B., Department of Veterinary Medicine and Surgery, the University of Texas MD Anderson Cancer Center, Houston, TX; of D.F., Vaccine and Infectious Disease Organization-International Vaccine Center, University of Saskatchewan, Saskatoon, SK, Canada. 
fatal pneumonia with acute respiratory distress syndrome occasionally accompanied by acute renal failure or gastrointestinal disease. ${ }^{5}$ Most patients present with a fever and respiratory symptoms, which rapidly progress to pneumonia. The most common respiratory symptoms are attributed to lower respiratory tract disease and include dyspnea and coughing. ${ }^{6}$ Few individuals solely develop mild upper respiratory tract symptoms, such as a sore throat. ${ }^{6,7}$ Severe disease, and death, because of MERS-CoV infection is most common in individuals affected by comorbidities, including diabetes, renal or cardiac disease, and hypertension. ${ }^{8}$ The current case fatality rate is approximately $36 \%$ (World Health Organization, http://www.who.int/csr/don/30-september-2015mers-saudi-arabialen, last accessed October 9, 2015); however, no autopsy reports detailing the gross or histological lesions that develop in fatal human infections have been published to date. To elucidate the pathogenesis of this virus and investigate underlying mechanisms for the variation in disease severity seen in humans, two nonhuman primate models of MERS-CoV disease were developed. These models simulated the wide range of disease severity seen in infected humans. After MERS-CoV inoculation, rhesus macaques developed mild to moderate disease, whereas common marmosets exhibited moderate to severe, potentially lethal, disease. ${ }^{9,10}$

Clinical description and virology of MERS-CoV infection in the rhesus macaque and common marmoset models have been reported separately. ${ }^{9,10}$ Herein, we focus on detailed and specific histopathology aspects of the respiratory tract of infected animals to better define the pathology of MERS-CoV infection in the lungs. To this end, we quantitatively analyzed the bronchointerstitial pneumonia that developed in both nonhuman primate species after MERS-CoV inoculation and quantified the amount of MERS-CoV antigen in the lungs using digital imaging and analysis. We observed differences in pulmonary neutrophil infiltration and presence of viral antigen in rhesus macaques compared with common marmosets. Increased numbers of neutrophils in the lung and higher amounts of MERS-CoV antigen were observed in marmosets. However, marmosets and macaques had similar pulmonary expression of the MERS-CoV receptor, dipeptidyl peptidase 4 (DPP4). These results suggest that increased pulmonary virus replication and a robust local immune response to MERS-CoV infection may play a role in pulmonary pathology severity, with higher viral loads and a more pronounced acute inflammatory response observed in marmosets.

\section{Materials and Methods}

\section{Ethics and Biosafety Statements}

All animal experiments were approved by the Rocky Mountain Laboratories (RML; Hamilton, MT) Institutional Animal Care and Use Committee and were performed following the guidelines of the Association for Assessment and Accreditation of Laboratory Animal Care, International, by certified staff in an Association for Assessment and Accreditation of Laboratory Animal Care, International-approved facility. All infectious work with MERS-CoV was approved by the Institutional Biosafety Committee and performed in a high containment facility at RML. Sample inactivation was performed according to standard operating procedures approved by the Institutional Biosafety Committee for removal of specimens from high containment.

\section{Nonhuman Primates}

Archived tissue blocks from eight rhesus macaques (four males and four females; aged 4 to 10 years) inoculated with a total dose of $7 \times 10^{6} 50 \%$ tissue culture infectious dose of MERS-CoV and seven common marmosets (seven males; aged 2 to 6 years) inoculated with a total dose of $5.2 \times 10^{6}$ $50 \%$ tissue culture infectious dose of MERS-CoV, as described previously, ${ }^{9-12}$ were analyzed histologically. The rhesus macaques (RMs 1 to 8) and common marmosets (CMs 1 to 7) were randomly assigned a number. Necropsies of the animals were scheduled for 3 days after inoculation (dpi; CMs 1 to 3 and RMs 1 to 6) and 6 dpi (CMs 4 to 6 and RMs 7 to 8). The remaining common marmoset (CM7) was not originally scheduled for euthanasia; instead, it was to be used to study long-term survival. However, because of development of severe clinical signs, this animal and CM5 were euthanized 4 dpi. A complete set of tissues from each animal was collected at necropsy.

\section{Histopathology and IHC}

Histopathology and immunohistochemistry (IHC) were performed on rhesus macaque and common marmoset tissues. Tissues were fixed according to standard operating procedures for a minimum of 7 days in $10 \%$ neutral-buffered formalin, embedded in paraffin, and stained with $\mathrm{H} \& \mathrm{E}$.

IHC with a rabbit polyclonal antiserum against $\mathrm{HCoV}$ EMC/2012 (1:1000; RML) ${ }^{12}$ as a primary antibody was used to detect MERS-CoV antigen. IHC was further used to detect neutrophils (polyclonal goat anti-myeloperoxidase, 1:450; R\&D Systems, Minneapolis, MN), T cells (monoclonal rabbit anti-CD3, prediluted; Ventana, Tucson, AZ), B cells (polyclonal rabbit anti-CD20, 1:100; Thermo Scientific, Waltham, MA), macrophages (polyclonal rabbit anti-Iba1, 1:1000; RML), epithelial cells (polyclonal rabbit anti-pan cytokeratin, 1:50; Novus Biologicals, Littleton, CO), and DPP4 (polyclonal rabbit anti-DPP4/CD26, 1:100; LifeSpan BioSciences, Inc., Seattle, WA). DPP4 was labeled purple using the Discovery Purple kit (Ventana).

Sections of lung from animals necropsied 3 or 6 dpi that were labeled for MERS-CoV antigen or inflammatory cell markers were digitized using an Aperio Digital Slide Scanner (Leica, Wetzler, Germany) and analyzed using the positive pixel count algorithm in ImageScope version 


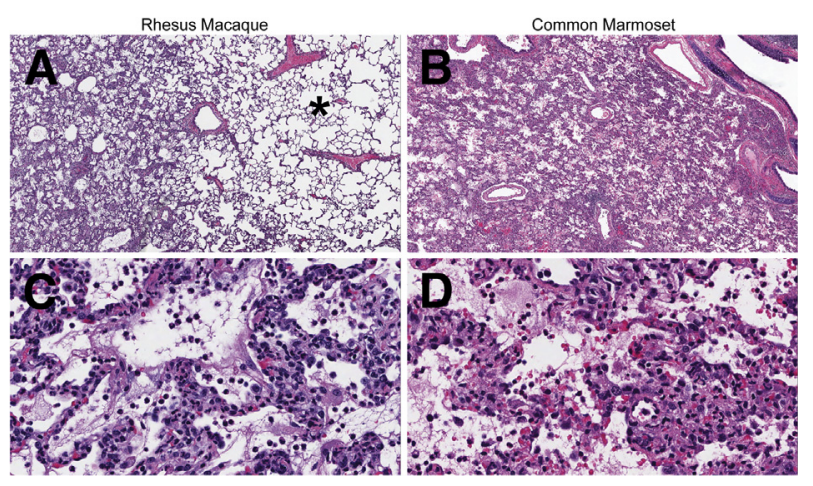

Figure 1 Middle East respiratory syndrome coronavirus-inoculated nonhuman primates develop bronchointerstitial pneumonia that is histologically similar in character, but is more extensive, in common marmosets. A-D: Representative sections of lung from a rhesus macaque ( $\mathbf{A}$ and $\mathbf{C}$ ) and common marmoset (B and $\mathbf{D}$ ) euthanized 3 days after inoculation. A: Unaffected pulmonary tissue (asterisk) adjacent to a focus of bronchointerstitial pneumonia. B: The lung is diffusely affected by bronchointerstitial pneumonia. C and D: The microscopic features of the bronchointerstitial pneumonia are similar in rhesus macaques and common marmosets. Alveolar septa and lumina are predominantly infiltrated by neutrophils and macrophages mixed with fibrin, hemorrhage, and edema. Hematoxylin and eosin staining was used. Original magnifications: $\times 4$ (A and B); $\times 40$ (C and D).

12.1.0.5029 (Leica). The ImageScope positive pixel count algorithm quantified the percentage of the pulmonary tissue that was positively labeled for MERS-CoV antigen or a specific type of inflammatory cell and the percentage of pulmonary tissue that did not express the IHC marker of interest, but which was labeled by a background stain. Positive pixel count algorithm calculations are on the basis of the amount of a specific stain present in a digitized slide and do not include non-stained areas, such as spaces filled with air. The lung lobe section that was most severely affected by bronchointerstitial pneumonia was analyzed in each animal.

\section{Statistical Analysis}

Statistical analyses were performed using the unpaired $t$-test. $P<0.05$ was considered statistically significant. Statistical analysis of data from 6 dpi was not always possible because there were only two animals remaining at this time point. All statistics were performed using GraphPad Prism version 6.02 (GraphPad Software, Inc., La Jolla, CA).

\section{Results}

Widespread Bronchointerstitial Pneumonia Develops in Common Marmosets

Macaques and marmosets developed bronchointerstitial pneumonia that predominantly centered on terminal bronchioles. $^{9-12}$ More detailed histological analysis revealed that in rhesus macaques the pulmonary lesions ranged from mild to severe; however, even in lung lobes with severe lesions, the lesions were multifocal and often surrounded by large areas of normal intervening lung tissue (Figure 1, A and C). The bronchointerstitial pneumonia in the common marmosets was of moderate to marked severity and was multifocal to coalescing, with some lobes diffusely affected (Figure 1, B and D). At both 3 and 6 dpi, the bronchointerstitial pneumonia was more severe in marmosets than in macaques. The more severe bronchointerstitial pneumonia that developed in common marmosets fit with the more severe respiratory clinical signs and more extensive pulmonary gross pathology that have previously been reported in common marmosets compared with rhesus macaques. $^{9-12}$

\section{Pulmonary Multinucleated Cells Are Predominantly of Macrophage Origin}

In both nonhuman primate species, the bronchointerstitial pneumonia was accompanied by multinucleated cells that were scattered within alveoli or that appeared to line the surface of alveolar septa. The multinucleated cells were present in macaques and marmosets necropsied on 3, 4, and 6 dpi. IHC for Ibal (Figure 2, A and B) and pan cytokeratin (Figure 2, C and D) on sections of lung tissue demonstrated that the multinucleated cells were a mixed population of cells. More than $80 \%$ of the multinucleated cells in macaques and marmosets expressed Iba1, indicating they were of macrophage origin; epithelial syncytia that expressed pan cytokeratin made up the remainder of the multinucleated cells.

\section{Airway Lesions Are More Severe in Common Marmosets}

The lesions that developed in bronchi and bronchioles in common marmosets necropsied 3, 4, or 6 dpi were more

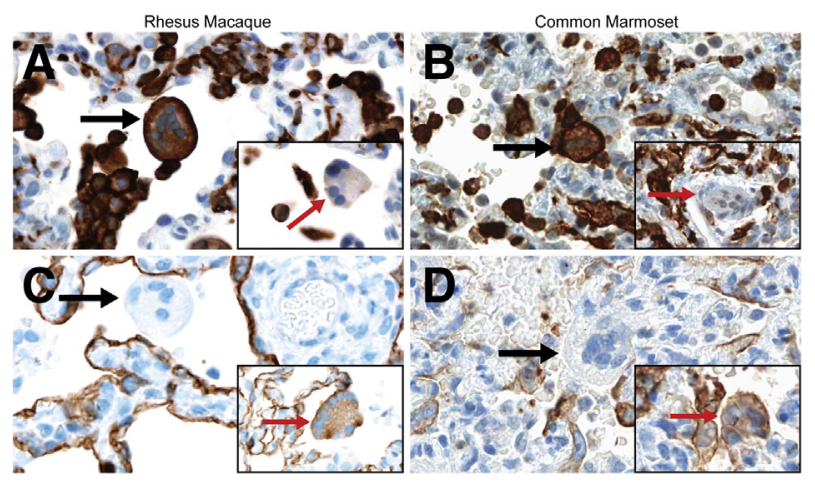

Figure 2 A mixed population of multinucleated cells are widely scattered throughout the bronchointerstitial pneumonia in rhesus macaques ( $A$ and $\mathbf{C}$ ) and common marmosets (B and $\mathbf{D}$ ). $\mathbf{A}$ and $\mathbf{B}$ : Immunohistochemistry (IHC) for Iba1 in sections of lung. Most of the multinucleated cells express Iba1 (black arrows), indicating the cells are of macrophage origin. Insets: Multinucleated cells that are not macrophages (red arrows), as indicated by their lack of Iba1 expression. C and D: IHC for pan cytokeratin in sections of lung. Most of the multinucleated cells are not of epithelial origin and do not express pan cytokeratin (black arrows). Insets: Multinucleated cells expressing pan cytokeratin (red arrows), indicating the cells are of epithelial origin. Original magnification, $\times 40$ (main images and insets). 


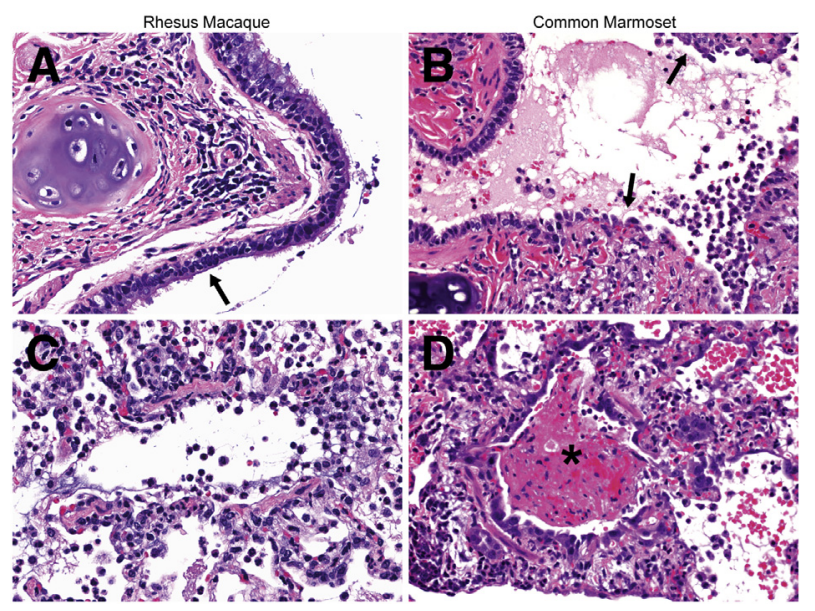

Figure 3 Middle East respiratory syndrome coronavirus-inoculated common marmosets develop more severe airway lesions than rhesus macaques. A: Respiratory epithelium in a bronchus exhibits focal loss of cilia (arrow) in a macaque 3 days after inoculation (dpi). Rare inflammatory cells are present in the bronchial lumen. B: Respiratory epithelial cells in a bronchus are eroded and attenuated (arrows) in a marmoset 3 dpi. Neutrophils and foamy macrophages infiltrate the bronchial wall and mix with edema and hemorrhage in the bronchial lumen. C: Neutrophils and foamy macrophages with minimal edema, hemorrhage, and fibrin are present in the wall and lumen of a bronchiole in a macaque $3 \mathrm{dpi}$. D: A bronchiole is occluded by a mat of fibrin (asterisk) mixed with edema, hemorrhage, and degenerate leukocytes in a marmoset $4 \mathrm{dpi}$. Hematoxylin and eosin staining was used. Original magnification, $\times 40$ (A-D).

severe than those observed in rhesus macaques. Compared with the marmosets, airway lesions in macaques were mild. The respiratory epithelium that lined bronchi was rarely damaged in macaques; when epithelial lesions were present, mild respiratory epithelial degeneration with loss of cilia was observed (Figure 3A). Multiple bronchioles in macaques were mildly infiltrated by neutrophils with fewer macrophages (Figure 3C). Occlusion of bronchioles by large accumulations of fibrin was not observed in macaques at any time point. At all time points, affected bronchi and bronchioles in common marmosets were multifocally eroded and lined by attenuated respiratory epithelium that lacked cilia (Figure 3B). Airways were often infiltrated predominantly by neutrophils and macrophages mixed with varying amounts of fibrin, edema, and hemorrhage (Figure 3D).

\section{Higher Amounts of Pulmonary Viral Antigen Are Detected in Common Marmosets}

MERS-CoV antigen was detected by IHC in sections of lung from marmosets and macaques necropsied 3 or 6 dpi (Figure 4, A and B). In both nonhuman primate species, MERS-CoV antigen was detected predominantly in type I and type II pneumocytes and was occasionally identified in macrophages (Figure 4, C and D). The percentage of the lung positively labeled for MERS-CoV antigen was quantified by the ImageScope positive pixel count algorithm (Figure 4E). At both 3 and $6 \mathrm{dpi}$, common marmosets had a higher mean percentage of the lung positively labeled for MERS-CoV antigen than rhesus macaques. At 3 dpi, $14.5 \%$ of the pulmonary parenchyma in marmosets contained viral antigen, significantly higher than the $3.6 \%$ detected in the lungs of rhesus macaques $(P=0.030)$. Although statistics could not be performed at 6 dpi because there were only two animals at this time point, a higher percentage of the marmoset lung still labeled positive for viral antigen than the rhesus macaque
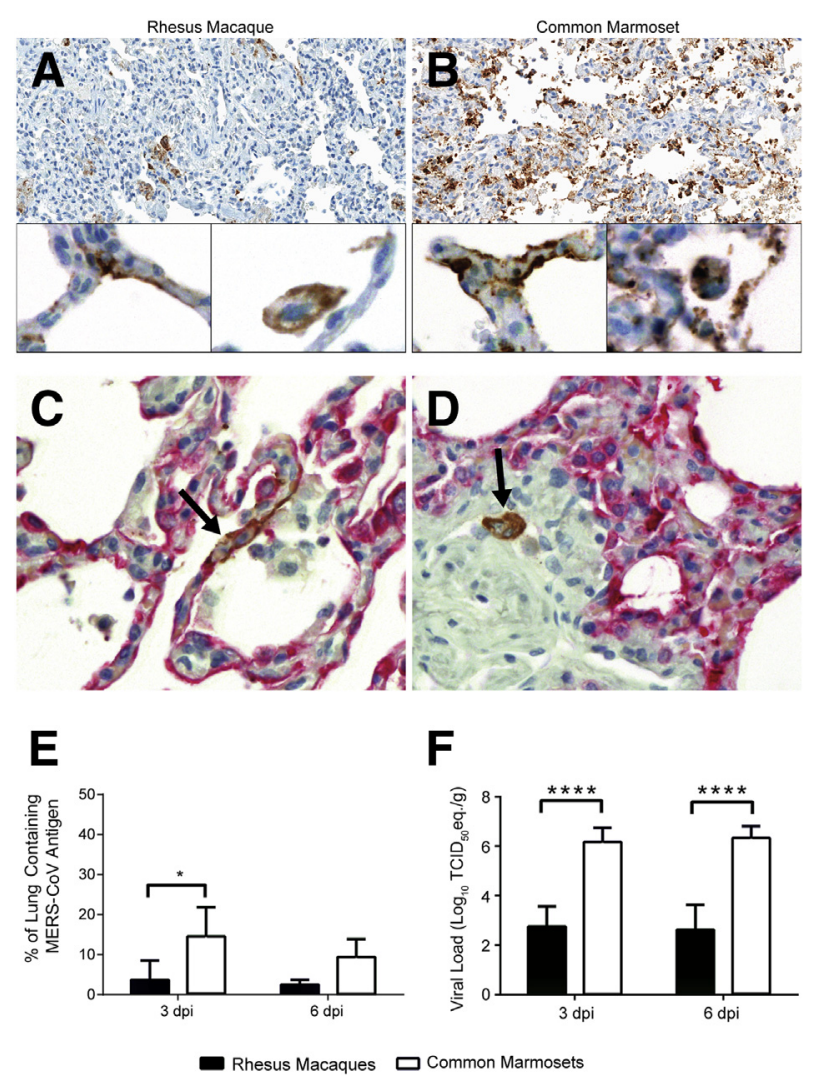

Figure 4 Common marmoset lungs contain more Middle East respiratory syndrome coronavirus (MERS-CoV) antigen than rhesus macaque lungs. A and B: Immunohistochemistry (IHC) for MERS-CoV antigen (labeled brown) in sections of lung from nonhuman primates necropsied 3 days after inoculation (dpi). Lower amounts of viral antigen are present in macaques (A) than marmosets (B). At higher magnification, viral antigen is seen in pneumocytes (left insets) and in macrophages (right insets). C and D: IHC for pan cytokeratin (labeled red) and MERS-CoV antigen (labeled brown) in the lung from a rhesus macaque necropsied 3 dpi. C: Viral antigen is present in the cytoplasm of a pneumocyte (arrow), as identified by the morphology of the cell and its expression of pan cytokeratin. D: Viral antigen is shown in a macrophage (arrow), as identified by its cellular morphology and lack of pan cytokeratin expression. E: The percentage of the lung containing MERS-CoV antigen is higher in common marmosets at both 3 and $6 \mathrm{dpi}$, as determined by digital analysis using ImageScope. Statistics could not be performed for the 6 dpi data because there were only two animals per time point. F: Pulmonary viral RNA loads are significantly higher in marmosets at both 3 and 6 dpi. ${ }^{*} P<0.05$, $\star * * * P<0.0001$ for rhesus macaques versus common marmosets. Original magnifications: $\times 20(\mathbf{A}$ and $\mathbf{B}) ; \times 40$ (insets, $C$ and $\mathbf{D})$. $\mathrm{TCID}_{50}, 50 \%$ tissue culture infectious dose. 

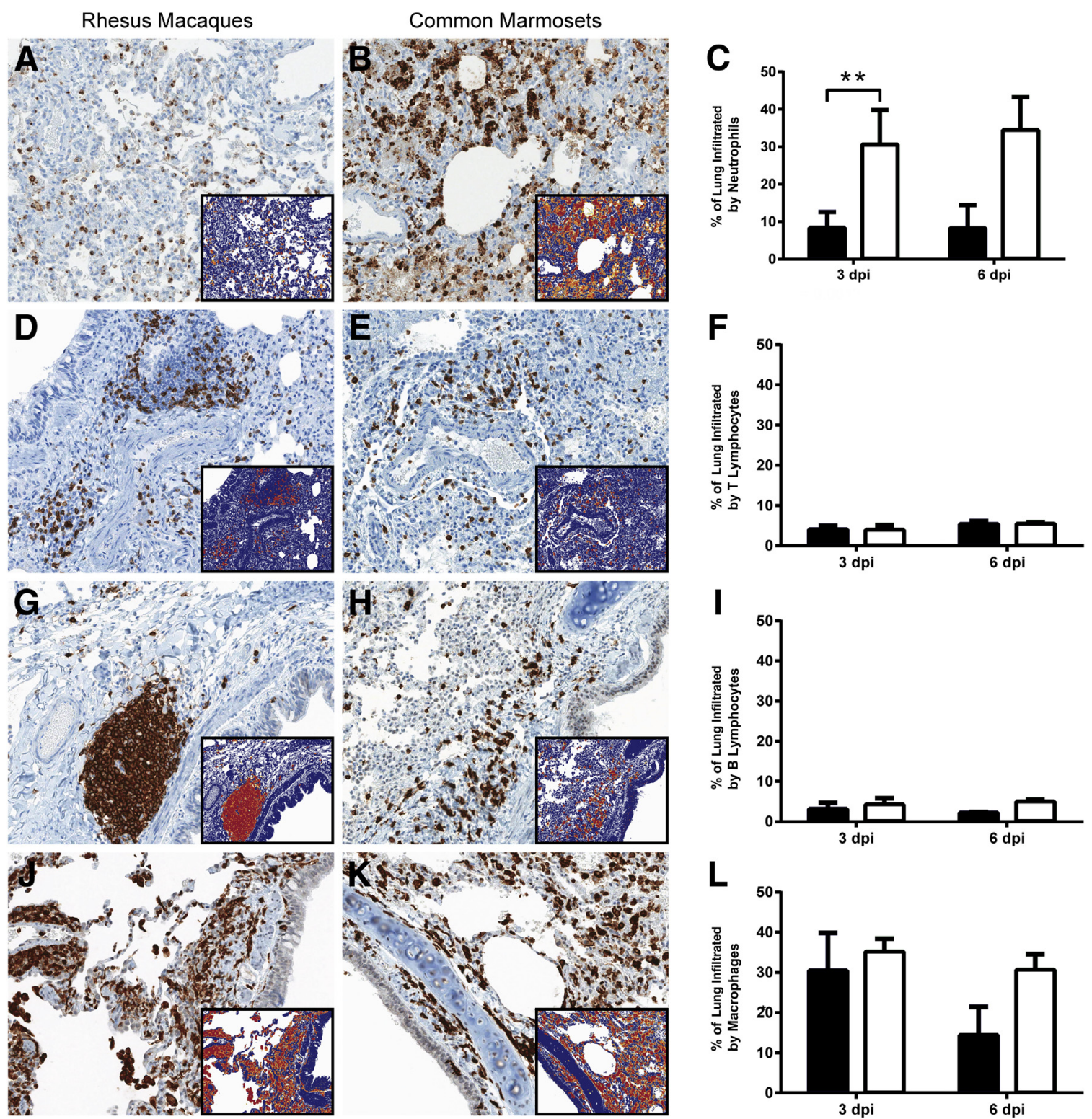

Figure 5 Quantification of inflammatory cells in the lung indicates that marmosets (white bars) exhibit higher pulmonary inflammatory cell infiltration at both 3 and 6 days after inoculation (dpi) compared with rhesus macaques (black bars). A and B: Immunohistochemistry for myeloperoxidase, a marker for neutrophils, in lung sections at $3 \mathrm{dpi}$. C: The percentage of the lung infiltrated by neutrophils is significantly higher in marmosets at 3 dpi. No statistically significant differences are noted for pulmonary infiltration by $\mathbf{T}$ lymphocytes $(\mathbf{D}-\mathbf{F})$, B lymphocytes $(\mathbf{G}-\mathbf{I})$, or macrophages $(\mathbf{J}-\mathbf{L})$ between macaques and marmosets at $3 \mathrm{dpi}$. The difference in pulmonary infiltration by neutrophils, B lymphocytes, and macrophages in common marmosets, compared with rhesus macaques, is greater at 6 than at $3 \mathrm{dpi}$. Statistics could not be performed for the 6 dpi data because there were only two animals per time point. Insets: The results of the ImageScope positive pixel count algorithm on the $3 \mathrm{dpi}$ immunohistochemically labeled lung sections. Red and orange pixels indicate detection of specific inflammatory cell markers; cells not expressing the marker of interest are shown as blue pixels. ${ }^{*} P<0.01$ for rhesus macaques versus common marmosets. Original magnification, $\times 20$ (main images and insets).

lung at this time point $(9.3 \%$ versus $2.4 \%)$. The results from the quantification of pulmonary MERS-CoV antigen fit with previously reported pulmonary viral RNA loads detected by quantitative RT-PCR. Retrospective pooling and reanalysis of pulmonary viral RNA load data from rhesus macaque ${ }^{10,12}$ and common marmoset lung tissues ${ }^{9}$ show that at 3 and $6 \mathrm{dpi}$, common marmosets had significantly higher pulmonary viral RNA loads $(P<0.0001)$, which were up to 1000 times higher than rhesus macaques necropsied at the same time point (Figure 4F).

\section{Pulmonary Neutrophil Infiltration Is Significantly Higher in Common Marmosets}

IHC was performed on sections of lung from marmosets and macaques necropsied at 3 and 6 dpi to detect neutrophils, $\mathrm{T}$ lymphocytes, B lymphocytes, and macrophages. The 


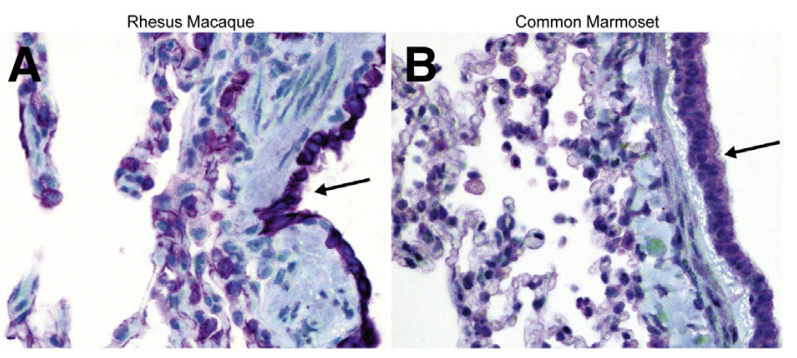

Figure 6 Dipeptidyl peptidase 4 (DPP4) is expressed by the same cell types in rhesus macaques and common marmosets in the lung. Immunohistochemistry for DPP4 on sections of lung show DPP4 is expressed by airway epithelium (arrows) and pneumocytes in macaques $(\mathbf{A})$ and marmosets (B). DPP4 was labeled purple using the Discovery Purple kit; tissues were counterstained with hematoxylin. Original magnification, $\times 40$ (A and B).

percentage of the pulmonary section that was positively labeled for each of these specific inflammatory cell types was quantified by ImageScope (Figure 5, A-L). The mean percentage of the lung infiltrated by neutrophils, as detected by myeloperoxidase IHC, was significantly higher $(P<0.001)$ in marmosets than macaques at $3 \mathrm{dpi}$, with $30.5 \%$ of the marmoset lung lobes infiltrated by neutrophils compared with $8.3 \%$ of the macaque lung lobes. In both nonhuman primate species, neutrophils were abundant in alveolar lumina, with fewer neutrophils in airways, alveolar septa, and blood vessels. At $3 \mathrm{dpi}$, the mean percentage of the lung infiltrated by $\mathrm{T}$ lymphocytes, B lymphocytes, or macrophages in rhesus macaques compared with common marmosets was similar $(P>0.05)$. In both nonhuman primate species, $\mathrm{T}$ and $\mathrm{B}$ lymphocytes exhibited segmental to circumferential cuffing of blood vessels, bronchi, and bronchioles, were widely scattered within alveolar septa and blood vessels, and were rarely present in alveolar lumina or airways (Supplemental Figure S1, A-D). Increased numbers of $\mathrm{T}$ and $\mathrm{B}$ lymphocytes were present in thickened alveolar septa compared with alveolar septa of normal width.

In rhesus macaques, multiple lymphoid follicles were present adjacent to bronchi or bronchioles. Lymphoid follicles were rarely observed in common marmosets; when present, lymphoid follicles developed near bronchi. In both species, lymphoid follicles were composed of centrally located B lymphocytes cuffed by $\mathrm{T}$ lymphocytes with variable numbers of macrophages scattered among the $\mathrm{B}$ and $\mathrm{T}$ lymphocytes.

In macaques and marmosets, numerous macrophages were identified within alveolar lumina and septa, whereas fewer macrophage-cuffed vascular walls and airways were detected within airways and vascular lumina (Supplemental Figure S1, E and F). The mean percentage of the lung infiltrated by macrophages was higher than the pulmonary infiltration by $\mathrm{T}$ or B lymphocytes in both macaques and marmosets.

At $6 \mathrm{dpi}$, the mean percentage of the lung infiltrated by neutrophils was higher in marmosets than macaques (34.4\% versus $8.2 \%$ ) (Figure $5 \mathrm{C}$ ). The mean percentage of the lung infiltrated by $\mathrm{T}$ lymphocytes was similar in marmosets and macaques (Figure 5F). Although statistics could not be performed on the 6 dpi data because there were only two animals per time point, the percentage of the lung infiltrated by B lymphocytes (4.9\% versus $2.2 \%$ ) and macrophages $(30.6 \%$ versus $14.3 \%)$ was higher in marmosets than macaques (Figure 5, I and L). The location of the inflammatory cell types at 6 dpi was similar to that described at 3 dpi.

\section{DPP4 Is Expressed by Similar Cell Types in the Lungs of Rhesus Macaques and Common Marmosets}

To determine whether the difference in lesion severity between common marmosets and rhesus macaques could be explained by a difference in expression of the receptor for MERS-CoV, IHC for DPP4 was performed on lung sections from each species (Figure 6, A and B). In both rhesus macaques and common marmosets, DPP4 was shown to be expressed by pneumocytes, airway epithelium, smooth muscle cells, endothelium, and macrophages. Visually, similar percentages of each cell type expressed DPP4 in common marmosets compared with rhesus macaques. In both species, MERS-CoV antigen was detected in several of the cell types that expressed DPP4 in the lung, including type I and type II pneumocytes and alveolar macrophages.

\section{Discussion}

Epidemiological data have shown marked variation in clinical disease severity in humans infected with MERS$\mathrm{CoV} .{ }^{5,6,13}$ This article details the differences and similarities in pulmonary lesion severity, influx of inflammatory cells into the lungs, and pulmonary viral antigen and RNA loads in two nonhuman primate models of MERS-CoV infection exhibiting mild versus severe disease.

A mixed population of multinucleated cells was observed in areas of bronchointerstitial pneumonia in macaques and marmosets. The multinucleated cells were predominantly of macrophage origin, whereas the remainder was of epithelial origin. Other coronaviruses, including severe acute respiratory syndrome coronavirus, which causes pneumonia in humans, have been associated with the development of multinucleated cells of macrophage or epithelial origin. ${ }^{14,15}$ Viral-induced cell-to-cell fusion may have caused the formation of the mixed population of multinucleated cells in the MERS$\mathrm{CoV}-$ inoculated macaques and marmosets. MERS-CoV antigen was detected in the cytoplasm of scattered macrophages in marmoset and macaque lungs using IHC. Although it is possible that the viral antigen present in some of the macrophages was because of phagocytosis of cellular debris containing the virus, human macrophages can be productively infected with MERS-CoV,${ }^{16}$ which 
may have resulted in the formation of multinucleated giant cells in the macaques and marmosets.

Bronchointerstitial pneumonia developed in both rhesus macaques and common marmosets after MERS-CoV inoculation; however, the percentage of the lung affected by lesions and infiltrated by neutrophils was higher in marmosets than in macaques at both 3 and 6 dpi. The higher pulmonary viral loads observed in the common marmosets at both 3 and 6 dpi may have induced a more robust acute inflammatory response, resulting in increased neutrophil recruitment to the lungs. Once present in the lungs, neutrophils can degranulate or release reactive oxygen species extracellularly, damaging pulmonary tissue and potentially causing more extensive pulmonary lesions and increased clinical disease severity. ${ }^{17,18}$

At 3 dpi, no differences were detected for T-lymphocyte, B-lymphocyte, or macrophage infiltration into the lungs of marmosets compared with macaques. However, upregulation of genes or RNA transcripts associated with proinflammatory mediators has been shown in areas of the lung affected by pneumonia at 3 dpi in both species. ${ }^{9,10}$ These results fit with what would be expected in tissues during the early phase of inflammation when an innate localized immune response is induced and neutrophils are the predominant effector cell type, before activation of the adaptive immune response. ${ }^{19,20}$ Marked changes in the numbers of infiltrating lymphocytes and macrophages usually are not evident until the later stages of inflammation. By $6 \mathrm{dpi}$, the difference in the mean percentage of the lung infiltrated by neutrophils, B lymphocytes, and macrophages had increased in marmosets compared with macaques; however, the few animals necropsied at the 6 dpi time point prevented statistical analyses from being performed at this time point. In macaques, at $6 \mathrm{dpi}$, there was a decrease in the mean percentage of the lung infiltrated by $B$ lymphocytes and macrophages, which was associated with a decline in pulmonary viral antigen and viral loads. These results suggest that the viral infection was being cleared from the lung and that the inflammatory process was starting to resolve. At $6 \mathrm{dpi}$, the mean percentage of the lung infiltrated by neutrophils in rhesus macaques was similar to that at $3 \mathrm{dpi}$. However, in common marmosets, there was an increase in pulmonary neutrophil influx between 3 and 6 dpi, suggesting that there was ongoing acute pulmonary damage with resultant continued recruitment of neutrophils to the lung.

At 3 and 6 dpi, higher pulmonary viral antigen and viral RNA loads were detected in common marmosets compared with rhesus macaques. The exact reason for the variation in virus replication rates in the lungs of these two nonhuman primate species is unknown. The difference in pulmonary viral loads was most likely not caused by differences in DPP4 expression, because the location and extent of DPP4 expression in the lungs was similar in the macaques and marmosets. The pulmonary viral load disparity may be because of differences between old world and new world primates in their susceptibility to MERS-CoV infection and virus replication, with rhesus macaques being less susceptible, or differences in the innate local immune response to a viral infection in the lung, which could lead to variations in MERS-CoV-induced disease severity. Differences in viral loads and virus replication or disease severity between old world and new world primates have been described for other viral infections. ${ }^{21-23}$ In addition, anatomical differences between the respiratory tracts of marmosets and macaques may also influence disease severity. Although the greater pulmonary viral load and its injurious effects on the pulmonary parenchyma are likely responsible for the increased inflammatory response observed in common marmosets, alternatively, it is possible that the more robust pulmonary inflammatory response in the marmosets may have promoted MERS-CoV replication, causing higher pulmonary viral loads in this primate species. It has previously been described that proinflammatory mediators and pathways can enhance replication of influenza $A$ virus and herpes simplex virus type $1^{24-26}$; similarly, MERS-CoV replication may be enhanced in a proinflammatory environment, resulting in the higher viral loads observed in common marmosets. The increased pulmonary neutrophil infiltration at both 3 and 6 dpi, rather than the pulmonary viral load alone, in the marmosets likely caused the increased extent of the pulmonary lesions, which led to the development of more severe clinical signs. These results suggest that increased virus replication, along with an intense local immune response to MERS-CoV infection, may result in the development of severe respiratory disease.

Overall, the comparison of these two nonhuman primate models has allowed us to better understand the pathogenesis of MERS-CoV infections and development of pulmonary lesions. In both species, we were able to detail changes in the influx of inflammatory cells in the lungs over time and show how the inflammatory process was associated with changes in pulmonary viral loads and viral antigen. Lower pulmonary viral loads and viral antigen in rhesus macaques were associated with a lower influx of neutrophils into the lung compared with common marmosets. In addition, we showed that differences in pulmonary viral loads and viral antigen between macaques and marmosets were not because of differences in pulmonary DPP4 expression.

The differences in MERS-CoV disease severity between rhesus macaques and common marmosets allow these two animal models to span the wide range of disease severity reported in MERS-CoV-infected humans. Although both nonhuman primate models can be used to investigate the pathogenesis of this disease, each model may be used for different applications. Rhesus macaques can serve as a model for mild MERS-CoV disease, which is increasingly being reported in humans infected with MERS-CoV. ${ }^{27,28}$ Common marmosets are the more suitable model for severe, potentially fatal, cases of MERS-CoV disease, which are typically reported in individuals who have an underlying comorbidity or in individuals of an older age. The severe bronchointerstitial pneumonia that develops in 
common marmosets inoculated with MERS-CoV makes marmosets an ideal model for testing the efficacy of medical countermeasures, such as antivirals, therapeutics, and vaccines. The smaller size of common marmosets favors this model for drug studies because it significantly lowers drug quantities; however, it precludes repeated blood sampling within a short time frame, and fewer species-specific reagents are available for marmosets compared with rhesus macaques. These limitations suggest that rhesus macaques may be a more suitable model for vaccine studies if repeated analysis of immune parameters is warranted. Although each model may be better suited for various applications, together, these two nonhuman primate models will aid in investigations aimed at combating the ongoing occurrence of human cases of MERS-CoV disease.

\section{Acknowledgments}

We thank Drs. Bart Haagmans and Ron Fouchier (Erasmus Medical Center, Rotterdamn, the Netherlands) for providing the MERS coronavirus isolate $\mathrm{HCoV}-\mathrm{EMC} / 2012$, Dan Long for his histopathology expertise, Ryan Kissinger and Austin Athman for editing figures, and all members of the Rocky Mountain Veterinary Branch (National Institute of Allergy and Infectious Diseases, NIH, Hamilton, MT) for their assistance.

\section{Supplemental Data}

Supplemental material for this article can be found at http://dx.doi.org/10.1016/j.ajpath.2015.10.025.

\section{References}

1. Zaki AM, van Boheemen $\mathrm{S}$, Bestebroer $\mathrm{TM}$, Osterhaus $\mathrm{AD}$, Fouchier RA: Isolation of a novel coronavirus from a man with pneumonia in Saudi Arabia. N Engl J Med 2012, 367:1814-1820

2. Al-Tawfiq JA, Memish ZA: Middle East respiratory syndrome coronavirus: epidemiology and disease control measures. Infect Drug Resist 2014, 7:281-287

3. Adney DR, van Doremalen N, Brown VR, Bushmaker T, Scott D, de Wit E, Bowen RA, Munster VJ: Replication and shedding of MERS$\mathrm{CoV}$ in upper respiratory tract of inoculated dromedary camels. Emerg Infect Dis 2014, 20:1999-2005

4. Reusken CB, Farag EA, Jonges M, Godeke GJ, El-Sayed AM, Pas SD, Raj VS, Mohran KA, Moussa HA, Ghobashy H, Alhajri F, Ibrahim AK, Bosch BJ, Pasha SK, Al-Romaihi HE, Al-Thani M, AlMarri SA, AlHajri MM, Haagmans BL, Koopmans MP: Middle East respiratory syndrome coronavirus (MERS-CoV) RNA and neutralising antibodies in milk collected according to local customs from dromedary camels, Qatar, April 2014. Euro Surveill 2014, 19:8-12

5. WHO MERS-CoV Research Group: State of knowledge and data gaps of Middle East respiratory syndrome coronavirus (MERSCoV) in humans. PLoS Curr 2013, 5. doi:10.1371/currents.outbreaks. 0bf719e352e7478f8ad85fa30127ddb8

6. Saad M, Omrani AS, Baig K, Bahloul A, Elzein F, Matin MA, Selim MA, Mutairi MA, Nakhli DA, Aidaroos AY, Sherbeeni NA, Al-Khashan HI, Memish ZA, Albarrak AM: Clinical aspects and outcomes of 70 patients with Middle East respiratory syndrome coronavirus infection: a single-center experience in Saudi Arabia. Int J Infect Dis 2014, 29:301-306

7. Assiri A, Al-Tawfiq JA, Al-Rabeeah AA, Al-Rabiah FA, Al-Hajjar S, Al-Barrak A, Flemban H, Al-Nassir WN, Balkhy HH, Al-Hakeem RF, Makhdoom HQ, Zumla AI, Memish ZA: Epidemiological, demographic, and clinical characteristics of 47 cases of Middle East respiratory syndrome coronavirus disease from Saudi Arabia: a descriptive study. Lancet Infect Dis 2013, 13:752-761

8. Arabi YM, Arifi AA, Balkhy HH, Najm H, Aldawood AS, Ghabashi A, Hawa H, Alothman A, Khaldi A, Al Raiy B: Clinical course and outcomes of critically ill patients with Middle East respiratory syndrome coronavirus infection. Ann Intern Med 2014, 160: 389-397

9. Falzarano D, de Wit E, Feldmann F, Rasmussen AL, Okumura A, Peng X, Thomas MJ, van Doremalen N, Haddock E, Nagy L, LaCasse R, Liu T, Zhu J, McLellan JS, Scott DP, Katze MG, Feldmann H, Munster VJ: Infection with MERS-CoV causes lethal pneumonia in the common marmoset. PLoS Pathog 2014, 10: e1004250

10. de Wit E, Rasmussen AL, Falzarano D, Bushmaker T, Feldmann F, Brining DL, Fischer ER, Martellaro C, Okumura A, Chang J, Scott D, Benecke AG, Katze MG, Feldmann H, Munster VJ: Middle East respiratory syndrome coronavirus (MERS-CoV) causes transient lower respiratory tract infection in rhesus macaques. Proc Natl Acad Sci U S A 2013, 110:16598-16603

11. Munster VJ, de Wit E, Feldmann H: Pneumonia from human coronavirus in a macaque model. N Engl J Med 2013, 368:1560-1562

12. Falzarano D, de Wit E, Rasmussen AL, Feldmann F, Okumura A, Scott DP, Brining D, Bushmaker T, Martellaro C, Baseler L, Benecke AG, Katze MG, Munster VJ, Feldmann H: Treatment with interferon- $\alpha 2 b$ and ribavirin improves outcome in MERS-CoVinfected rhesus macaques. Nat Med 2013, 19:1313-1317

13. Maltezou HC, Tsiodras S: Middle East respiratory syndrome coronavirus: implications for health care facilities. Am J Infect Control 2014, 42:1261-1265

14. Tse GM, To KF, Chan PK, Lo AW, Ng KC, Wu A, Lee N, Wong HC, Mak SM, Chan KF, Hui DS, Sung JJ, Ng HK: Pulmonary pathological features in coronavirus associated severe acute respiratory syndrome (SARS). J Clin Pathol 2004, 57:260-265

15. Kuiken T, Fouchier RA, Schutten M, Rimmelzwaan GF, van Amerongen G, van Riel D, Laman JD, de Jong T, van Doornum G, Lim W, Ling AE, Chan PK, Tam JS, Zambon MC, Gopal R, Drosten C, van der Werf S, Escriou N, Manuguerra JC, Stohr K, Peiris JS, Osterhaus AD: Newly discovered coronavirus as the primary cause of severe acute respiratory syndrome. Lancet 2003, 362: 263-270

16. Zhou J, Chu H, Li C, Wong BH, Cheng ZS, Poon VK, Sun T, Lau CC, Wong KK, Chan JY, Chan JF, To KK, Chan KH, Zheng BJ, Yuen KY: Active replication of Middle East respiratory syndrome coronavirus and aberrant induction of inflammatory cytokines and chemokines in human macrophages: implications for pathogenesis. J Infect Dis 2014, 209:1331-1342

17. Grommes J, Soehnlein O: Contribution of neutrophils to acute lung injury. Mol Med 2011, 17:293-307

18. Lacy P: Mechanisms of degranulation in neutrophils. Allergy Asthma Clin Immunol 2006, 2:98-108

19. Kolaczkowska E, Kubes P: Neutrophil recruitment and function in health and inflammation. Nat Rev Immunol 2013, 13:159-175

20. Amulic B, Cazalet C, Hayes GL, Metzler KD, Zychlinsky A: Neutrophil function: from mechanisms to disease. Annu Rev Immunol 2012, 30:459-489

21. Vargas-Mendez O, Elton NW: Naturally acquired yellow fever in wild monkeys of Costa Rica. Am J Trop Med Hyg 1953, 2:850-863

22. Smithburn KC, Haddow AJ: The susceptibility of African wild animals to yellow fever. Am J Trop Med Hyg 1949, 29:389-423

23. Verstrepen BE, Fagrouch Z, van Heteren M, Buitendijk H, Haaksma T, Beenhakker N, Palu G, Richner JM, Diamond MS, Bogers WM, 
Barzon L, Chabierski S, Ulbert S, Kondova I, Verschoor EJ: Experimental infection of rhesus macaques and common marmosets with a European strain of West Nile virus. PLoS Negl Trop Dis 2014, 8: e2797

24. Mogensen TH, Paludan SR: Molecular pathways in virus-induced cytokine production. Microbiol Mol Biol Rev 2001, 65:131-150

25. Pang IK, Pillai PS, Iwasaki A: Efficient influenza A virus replication in the respiratory tract requires signals from TLR7 and RIG-I. Proc Natl Acad Sci U S A 2013, 110:13910-13915

26. Patel A, Hanson J, McLean TI, Olgiate J, Hilton M, Miller WE, Bachenheimer SL: Herpes simplex type 1 induction of persistent
NF-kappa B nuclear translocation increases the efficiency of virus replication. Virology 1998, 247:212-222

27. Oboho IK, Tomczyk SM, Al-Asmari AM, Banjar AA, Al-Mugti H, Aloraini MS, Alkhaldi KZ, Almohammadi EL, Alraddadi BM, Gerber SI, Swerdlow DL, Watson JT, Madani TA: 2014 MERS-CoV outbreak in Jeddah-a link to health care facilities. N Engl J Med 2015, 372:846-854

28. Omrani AS, Matin MA, Haddad Q, Al-Nakhli D, Memish ZA, Albarrak AM: A family cluster of Middle East respiratory syndrome coronavirus infections related to a likely unrecognized asymptomatic or mild case. Int J Infect Dis 2013, 17:e668-e672 